\title{
PERFORMANCE ANALYSIS OF UPLINK ADAPTATION IN EUTRAN
}

\author{
Ankit Saxena* and Ravi Sindal*
}

\begin{abstract}
The evolution in mobile access technology improves radio resources utilization. In a wireless network, power constraints are observed at both links of communication, uplink (UL) towards the base station and downlink (DL) towards user equipment (UE). In DL, there are multiple sources of power available, but towards UL, there are limited power sources, that is, batteries. In this paper, we evaluate the performance of an open-loop and a closed-loop power control for LTE, through a comprehensive simulation with the help of key performance indicators (KPIs), and identify an optimum solution. We measure different KPIs such as inter-cell interference, signalto-interference noise ratio (SINR), reference signal received power (RSRP), throughput, spectral efficiency and power spectral density (PSD) for typical networks. As per our results, the performance of open-loop uplink power control (OLUPC) is better for an edge or boundary UE in comparison with closed-loop uplink power control (CLUPC). Furthermore, for the optimal solution in CLUPC, we tuned the value of the compensation factor $(\alpha)$ to meet the trade-off between high UL throughput and SINR. The conclusion of this work is that the best optimum solution can be obtained by selecting values of $\alpha$ near 0.95 for CLUPC and that this optimum solution gives better results in terms of good radio signal condition and UL throughput.
\end{abstract}

\section{Key Words}

LTE, link adaptation, EUTRAN, open-loop uplink power control (OLUPC), closed-loop uplink power control (CLUPC)

\section{Introduction}

The LTE EUTRAN uses orthogonal frequency-division multiple access as its radio access technology in uplink (UL) that eliminates mutual interference between users in the same cell; however, the interference from users in a neighbouring cell can affect the system performance, in terms of capacity and user throughput. For effective utilization, Third-Generation Partnership Project (3GPP) LTE [1] offers designed cell configuration with frequency

* Department of Electronics and Telecommunication, Institute of Engineering and Technology, Devi Ahilya Vishwavidyalaya (DAVV), Indore, India; e-mail: \{asaxena2014, rsindal\}@ietdavv.edu.in

Recommended by Dr. Leone C. Monticone

(DOI: 10.2316/Journal.214.2017.5.214-1081) reuse concept, where all cells in the network use the same frequency bands. Thus, complexity is created for effective resource utilization for both data and control channels at the inter-cell boundary.

In this paper, we create a similar complexity and tried to identify optimum solutions that would be of benefit for effective utilization of resources and enhance user throughput. This would adhere to our objective of obtaining a power control mechanism between user equipment (UE) and base station (eNB) that would play an important role in maintaining signal-to-interference noise ratio (SINR) at an acceptable level, near the inter-cell boundary. Two separate sources offer power to the respective links; from the UE transmitter called as UL and from the eNB transmitter called as Downlink (DL).

\subsection{Related Literature}

Authors in [2] have described an iterative algorithm with the help of user scheduling, data rate adaptation and power allocation for energy-efficient resource allocation. Authors in [3] state future challenges as link adaptation with a variety of channel state information such as capacity, signal and noise ratio (SNR), plus with different coding schemes such as a trellis, and space-time block coding. Authors in [4] concluded that inter-site distance plays an important role in the system as it affects the path loss and the SINR of the system. In [5], authors describe various ways for the resource allocations, methods of link adaptation (including power control and rate control), both with adaptive modulation and coding and hybrid automatic request. The authors also describe control-signalling encoding and channel state feedback for the purposes of link adaptation and resource allocation. Authors in [6], [7] show a significant improvement of CoMP in comparison with non-coordinated (N-CoMP) link adaptation in LTE-A, with interference. In addition, the authors concluded that the performance of CoMP is depending upon the channel imperfectness. In [8], the authors discuss an analysis of cell re-selection procedure followed in LTE and concluded that the SNR played an important role in channel allocation to UE.The authors in [9], [10] using system-level simulations compare the performance of five different media access control (MAC) scheduler under uniform LTE (EUTRAN) network in terms of key performance indicators (KPIs) for cell and UE. In [11], [12], authors discuss on the performance of 
proportional fair MAC scheduler over a feedback delay for EUTRAN.

\subsection{Key Performance Indicator}

\subsubsection{Interference for the Link}

It may be defined as the summation of all interfering signal power at the physical layer. This method is referred to as a Gaussian Interfering measurement method and is calculated in both directions, that is, UL and DL. It is used as input to measure SINR.

\subsubsection{Signal-to-Interference Noise Ratio DL}

The DL SINR $(\gamma)$ is the ratio of desirable DL power (eNB towards UE) for each radio block (RB) resource with all received signal power, including noise or interfering signal power (coming from another eNB link) on the same RB. It is expressed mathematically as follows:

$$
\gamma=\frac{\text { Power }_{\text {signal }}}{\text { Power }_{\text {signal }}+\Sigma \text { Power }_{\text {Interference }}}
$$

Similarly, the UL SINR is calculated for each RB. The radio link resource follows UE towards eNB.

\subsubsection{Reference Signal Received Power (RSRP)}

This is a metric to measure signal strength for a particular radio cell and is defined as the average power of the radio resource element within a $\mathrm{RB}$ measured in $\mathrm{dBm}$. It is used to identify the ranking of another neighbouring candidate cell by their signal strength. It can be expressed mathematically as follows:

$$
\begin{aligned}
R S R P & =\frac{\sum_{k=0}^{k-1} \frac{\sum_{m=0}^{M-1}(P(k, m))}{M}}{K} \\
& =\frac{\sum_{k=0}^{k-1}(P(k))}{K}
\end{aligned}
$$

- $P(k, m)$ represent the signal power of the radio element (RE) m within RB.

- $M$ is the number of REs carrying the RS in an RB. $K$ is the number of RBs.

\subsubsection{Throughput (UL and DL)}

It defined as the ratio of allocated transport block size with definite bandwidth per unit of time or TTI. Let $\mu$ be the TTI duration, $B$ be the transmission bandwidth configuration in a number of $\mathrm{RBs}, M$ be the modulation and coding scheme in use at the given SNR and $\mathrm{S}(M, B)$ the transport block size. The throughput $T$ in bit/s by each UE is calculated as follows:

$$
T=\frac{\mathrm{S}(M, B)}{\mu}
$$

\subsubsection{Spectral Efficiency (SE)}

It may be defined as the ratio of spectral resources utilized (SU) with spectral resources offered (SO). It is measured on the physical layer and is expressed in bit $/ \mathrm{s} / \mathrm{Hz}$ or bits per channel use (bpcu), equivalent to bit/symbol. Mathematically, we can evaluate as below:

$$
\mathrm{SE}=\mathrm{SU} / \mathrm{SO}
$$

While in LTE, we have a separate communication link between the UE and eNB, that is, UL and DL. For our analysis, we preferred to aggregate the throughput for all users in UL and DL split by the channel bandwidth to obtain SE.

\subsubsection{Power Spectral Density}

It defined as power availability in terms of amplitude at per unit frequency. It is used to measure a signal strength in the frequency domain. Mathematically, the PSD is computed from the spectrum Fast Fourier Transform of a signal. It helps us to measure channel impulse response per transmission time interval (TTI). It is affected by fading propagation conditions such as user's speed, channel taps, time granularity and frequency granularity. It is expressed in terms of $\mathrm{dBW} / \mathrm{Hz}$.

\subsection{Contribution}

This work yields new conclusions on UL power allocation procedure in practical systems, providing

1. A novel analysis for uplink performance with different KPIs.

2. An Optimized solution for Uplink Power Control at the cell boundary in terms of throughput and SINR.

\subsection{Outline}

Section 2 describes a method of UL power control in EUTRAN with LTE and a problem statement. Section 3 describes the implementation of link adaptation for the problem. Section 4 describes the performance analysis with respect to the optimum solution. The work is concluded in the last section.

\section{Link-Adaptation in LTE}

\subsection{Uplink Power Control Mechanism}

The 3GPP specifications define the setting of the UE transmit power, through physical uplink shared channel (PUSCH) measured in $\mathrm{dBm}$, as expressed below:

$P_{\mathrm{PUSCH}}=\min \left\{P_{\max }, 10 \cdot \log _{10} M+P_{\mathrm{o}}+\alpha \cdot \mathrm{PL}+\varphi_{\mathrm{mcs}}+f\left(\Delta_{i}\right)\right\}$

- $P_{\max }$ is the maximum UE transmit power, it depends on the UE power class.

- $M$ is the number of physical resource blocks (PRB) scheduled by the eNB. 
- $P_{\mathrm{o}}$ is a cell/UE-specific parameter signalled by radio resource control (RRC).

- $\alpha$ is the path loss compensation factor of a 3-bit cellspecific parameter in the range [ $\left[\begin{array}{ll}0 & 1\end{array}\right]$ managed by RRC.

- Path loss (PL) is the estimated DL path loss, calculated in the UE based on the reference symbol received power (RSRP).

- $m c s$ is a cell/UE-specific modulation and coding scheme as defined in the 3 GPP specifications for LTE. However, $\varphi_{\text {mcs }}$ is not included in our work.

- $f\left(\Delta_{i}\right)$ is UE specific. $\Delta_{i}$ is a closed-loop correction value and $f$ is a function that permits us to use the accumulated or absolute correction value.

$P_{\mathrm{o}}=\alpha \cdot\left(\mathrm{SNR}_{\mathrm{o}}+P_{\mathrm{n}}\right)+(1-\alpha)\left(P_{\max }-10 \cdot \log _{10}\left(M_{0}\right)\right)$

- $S N R_{\text {o }}$ is the open-loop SNR target.

- $P_{\mathrm{n}}$ is the noise power per PRB.

- $M_{0}$ defines the number of PRB s for which the SNR target is reached with full power. It is set to 1 in the implementation for simplicity.

$f\left(\Delta_{i}\right)$ is calculated by UE to determine the initial transmission power setting, based on parameters received from eNB and path loss. As $\Delta_{i}$ does not make a contribution to the initial power setting, we can modify (6) by ignoring $\varphi_{\mathrm{mcs}}$ and the closed-loop correction $f\left(\Delta_{i}\right)$ to obtain the following equation:

$$
P_{\mathrm{PUSCH}}=10 \cdot \log _{10} M+P_{\mathrm{o}}+\alpha \cdot \mathrm{PL}(\mathrm{dBm})
$$

In addition, as each PRB contains equal power, we can ignore power $M$ for each $\mathrm{PRB}$, resulting in the following:

$$
\mathrm{PSD}_{\mathrm{TX}}=P_{\mathrm{o}}+\alpha \cdot \mathrm{PL}(\mathrm{dBm} / \mathrm{PRB})
$$

For maintaining the balance of power throughout the cell, especially at a boundary or edge as per the $3 \mathrm{GPP}$ specification LTE offers fractional power enhancement called the $\alpha$ compensation factor for path loss. The value of $\alpha$ varies from 0 (no compensation) to 1 (full compensation) or its fractional (intermediate). We use the expression full compensation as a reference to the conventional power control method where the power is in the transmitter areas and is expressed by:

$$
P_{\mathrm{o}}=\mathrm{SNR}_{\mathrm{o}}+P_{\mathrm{n}}(\mathrm{dBm})
$$

Thus, PSD at the transmitting end, $\mathrm{PSD}_{\mathrm{TX}}$, is given by:

$$
\mathrm{PSD}_{\mathrm{TX}}=P_{\mathrm{o}}+\mathrm{PL}=\mathrm{SNR}_{0}+P_{\mathrm{n}}+\mathrm{PL}(\mathrm{dBm} / \mathrm{PRB})
$$

And the PSD at the receiving end, $\mathrm{PSD}_{\mathrm{RX}}$, is given by:

$$
\mathrm{PSD}_{\mathrm{RX}}=\left(\mathrm{SNR}_{\mathrm{o}}+P_{\mathrm{n}}\right)=P_{\mathrm{o}}(\mathrm{dBm} / \mathrm{PRB})
$$

On the other hand, to obtain the PSD for fractional power control at the transmitting end, $\mathrm{PSD}_{\mathrm{TX}}$, as given in (11), is modified to give:

$$
\begin{aligned}
\mathrm{PSD}_{\mathrm{TX}}= & P_{\mathrm{o}}+\alpha \mathrm{PL}=\alpha \cdot\left(\mathrm{SNR}_{\mathrm{o}}+P_{\mathrm{n}}\right)+(1-\alpha)\left(P_{\max }\right) \\
& +\alpha \mathrm{PL}(\mathrm{dBm} / \mathrm{PRB})
\end{aligned}
$$

Similarly, to obtain the PSD for fractional power control at the receiving end, $\mathrm{PSD}_{\mathrm{RX}}$, as given in (12), is modified to give:

$$
\mathrm{PSD}_{\mathrm{RX}}=P_{\mathrm{o}}+\mathrm{PL}(\alpha-1)(\mathrm{dBm} / \mathrm{PRB})
$$

In comparing (12) and (14), it should be noted that as the PSD in the conventional method is not a functional of PL, in the fractional method the term PL $(\alpha-1)$ is added. Here, we find that $P_{0}$ and $\alpha$ are the same for the network and only PL may change for UE towards a particular cell by the eNB.

\subsection{Problem Statement}

We define our problem under the following terms:

1. Comparative analysis between open-loop uplink power control (OLUPC) and closed-loop uplink power control (CLUPC) at the cell boundary or edge of the cell.

2. As per 3GPP specification, CLUPC is driven on the cell SINR, so we have to identify the optimum solution for UE at near eNB and at the cell boundary or edge.

3. In addition, we tried to optimize UL throughput performance by selecting values of $\alpha$ that satisfy SINR and UL throughput.

\section{Implementation of Link Adaptation}

\subsection{Experimental Setup}

For understanding, we have defined a typical system model of the LTE radio environment, including an eNB, UE, in association with the path loss model, the LTE spectrum model (including control and data) and other simulation parameters mentioned in Table 1. For proper analysis, we select three eNB values which are placed $1 \mathrm{~km}$ apart, forming three radio cells with a single UE as shown in Fig. 1. For testing and measurement, we use network simulator (NS-3).

Position of eNB are:

eNB 1: $x=0, y=0$; eNB 2: $x=1,000 \mathrm{~m}, y=0$; and eNB 3: $x=500, y=866$.

Position of UE are:

$\mathrm{UE}$ at the centre or near to the eNB are $x=0, y=0$; eNB 2: $x=1,000 \mathrm{~m}, y=0$; and eNB 3: $x=500, y=866$.

\subsection{Implementation of Uplink Power Control}

As described in Technical Specification 3Gpp TS36.213 section 5 [1], there are two types of UL power control mechanism: OLUPC and CLUPC are defined as:

1. In OLUPC, the UE transmission power depends on the estimation of the DL path loss and channel configuration.

2. In CLUPC, power control follows in OLUPC, with an addition of control by the eNB of the UE transmission power by means of explicit transmit power control commands transmitted in the DL. 
Table 1

Main Parameters Used in the Experiments

\begin{tabular}{|l|l|}
\hline Parameter & Configuration \\
\hline No. of UE & 1 \\
\hline No. of eNBs & 3 \\
\hline No. of bearers per UE & 3 \\
\hline Distance between eNB & $1,000 \mathrm{~m}$ \\
\hline Distance of UE from eNB & $0,660 \mathrm{~m}$ \\
\hline Speed of UE & $3 \mathrm{~km} / \mathrm{h}$ \\
\hline Base station power & $46 \mathrm{dBm}$ (effective isotropic radiated power) \\
\hline Carrier frequency & $2.0 \mathrm{GHz}$ \\
\hline Channel model & EVP model \\
\hline Mobility model & Constant positioning $(3 \mathrm{~km} / \mathrm{h})$ \\
\hline Fading model & Fading loss model \\
\hline Spectrum analyser & Resolution: $10 \mu \mathrm{s}$ \\
\hline Handover algorithm & A2A4 RSRQ \\
\hline eNB TX power & $30 \mathrm{dBm}$ \\
\hline UE TX power & $10 \mathrm{dBm}$ \\
\hline UE power control & Closed loop: enable \\
\hline Path loss model & Friis propagation loss model \\
\hline Epoch duration & $1 \mathrm{~s}$ \\
\hline Traffic type (UL + DL) & Voice and video (IP) \\
\hline Simulation time & $60 \mathrm{~s}$ \\
\hline
\end{tabular}

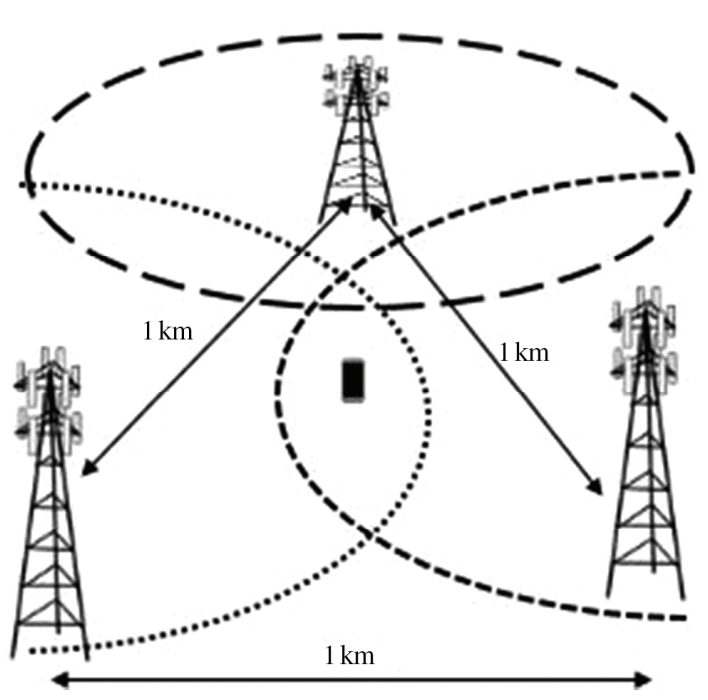

Figure 1. Illustration of LTE system model.

\section{Results}

As per simulation results, the OLUPC for UL offers better radio condition in comparison with the conventionally

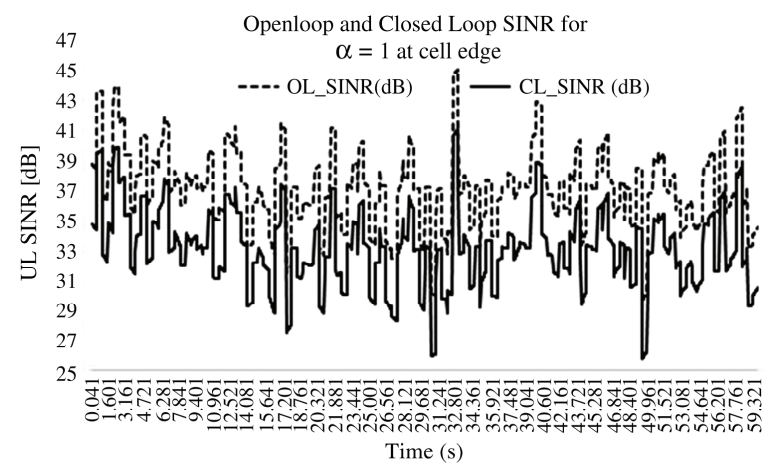

Figure 2. Illustration of UL SINR for OLUPC and CLUPC $\alpha=1$.

CLUPC as shown in Fig. 2. Here, we select $\alpha=1$ for both OLUPC and CLUPC.

As we observe, the performance of CLUPC for UL radio signal is inferior at the cell boundary and it is severely affected by neighbouring cell interference signal. In CLUPC, all users achieve equal SINR, resulting in lower throughput. The users at the cell edge are more affected by interference than the centre edge. It is to be noted that 


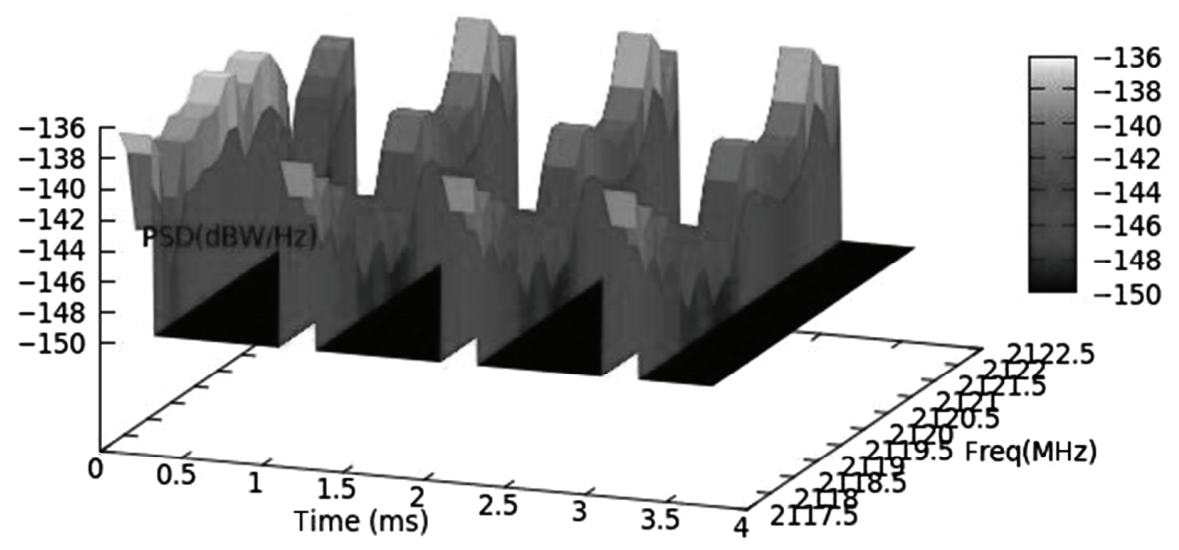

Figure 3. Illustration of measure PSD near to eNB.

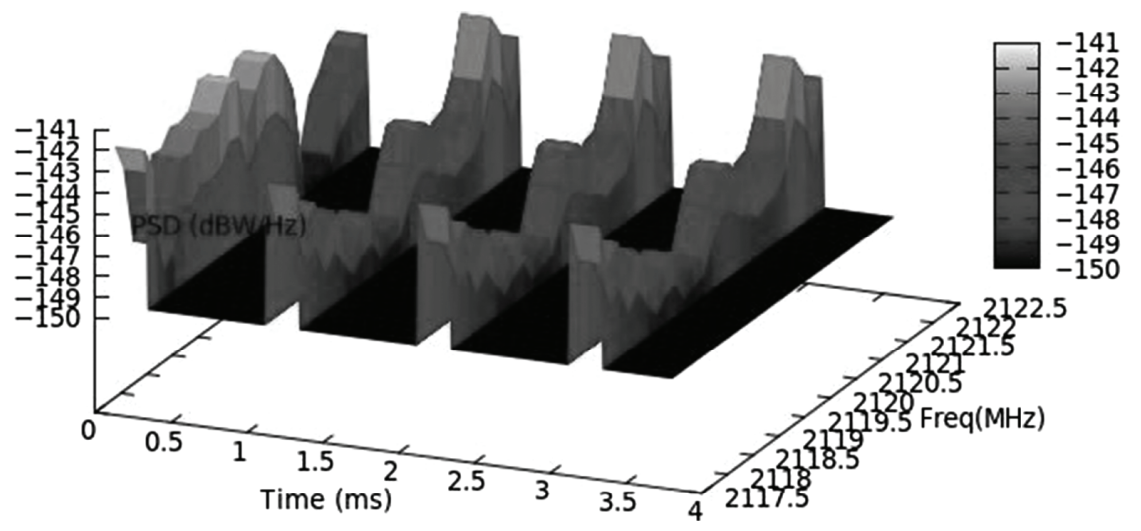

Figure 4. Illustration of measure PSD at the cell edge.

the CLUPC trade-off condition occurs between throughput and SINR at the cell edge. We measured PSD using spectrum analysers near to the eNB position, as shown in Fig. 3 and near the cell edge as shown in Fig. 4. We found that the difference of power density greater than $5-8 \mathrm{~dB}$ affects the cell boundary performance.

Figure 5 describes the SINR plot for the field, it shows the power level in $\mathrm{dB}$. We observe good values of SINR when the UE is near to eNB and as the UE moves away from the eNB the signal degrades. Also, we can find a degraded radio signal near to the edge boundary. Figure 6 describes simulation results for UL throughput for openloop and closed-loop power control in terms of $\alpha$. OLUPC performs well in comparison with CLUPC, as there is not any SINR condition to obey with OLUPC while in CLUPC a cell has to maintain throughout by a trade-off balance between different values of SINR, especially at the cell edge boundary. Figure 7 shows the UL performance for closed-loop power control of the centre and cell edge boundaries in terms of SINR and UL throughput rate. It is very clear that throughput depends upon the radio signal power. We have calculated SINR and throughput for different values of $\alpha$ under CLUPC, that is, $0<\alpha<1$. In observation, for $\alpha=0.95$ SINR, the difference between UE near to eNB with the UE at the cell boundary is less than $4 \mathrm{~dB}(\sim 3.95 \mathrm{~dB})$. That is the optimal balance for the

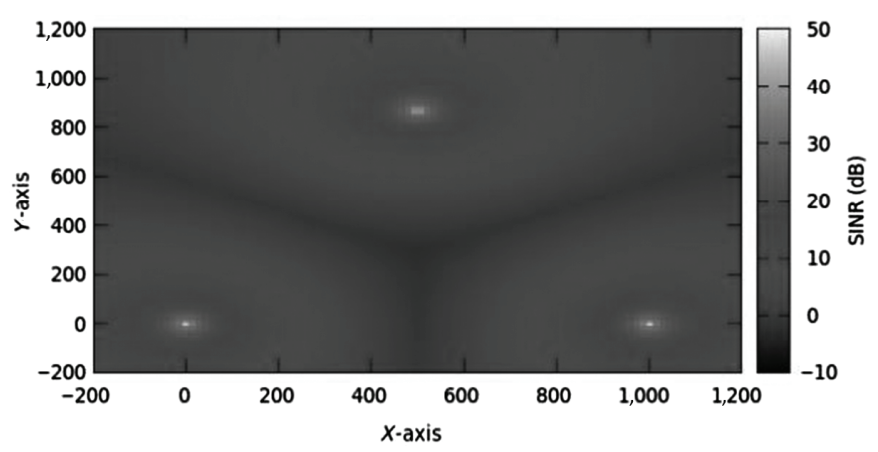

Figure 5. Illustration of measured SINR plot for experimental setup.

UE of the cell. Apart from that, for throughput of UE at the edge or boundary position, $\alpha=1$ gives a comparatively high throughput of $1.62 \mathrm{Mbps}$ which is $21 \%$ less than UE at the centre or near to the eNB location. While at $\alpha=0.95$, it gives a throughput of $1.03 \mathrm{Mbps}$ which is $48.9 \%$ lesser than UE at the centre or near to the location.

Furthermore, we select $\alpha=0.95$ as SINR of UL radio is better than SINR for $\alpha=1$. For $\alpha=1$, SINR of UL radio is $5.3 \mathrm{~dB}$ less with the SINR of UE at the centre or near to the eNB. This radio signal degradation is an effect of the higher system load while $\alpha$ at 0.95 loads gets distributed. 


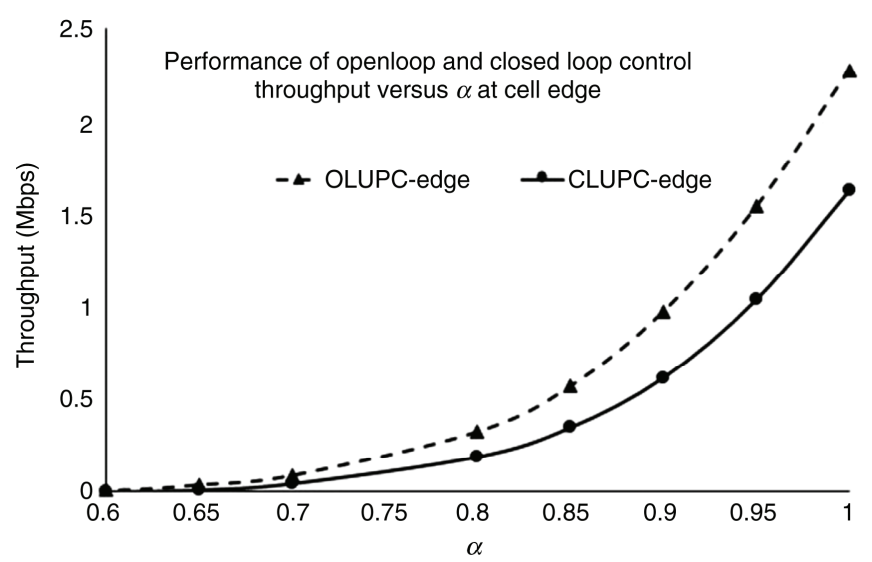

Figure 6. UL throughput for OLUPC and CLUPC with respect to $\alpha$ (compensation factor).

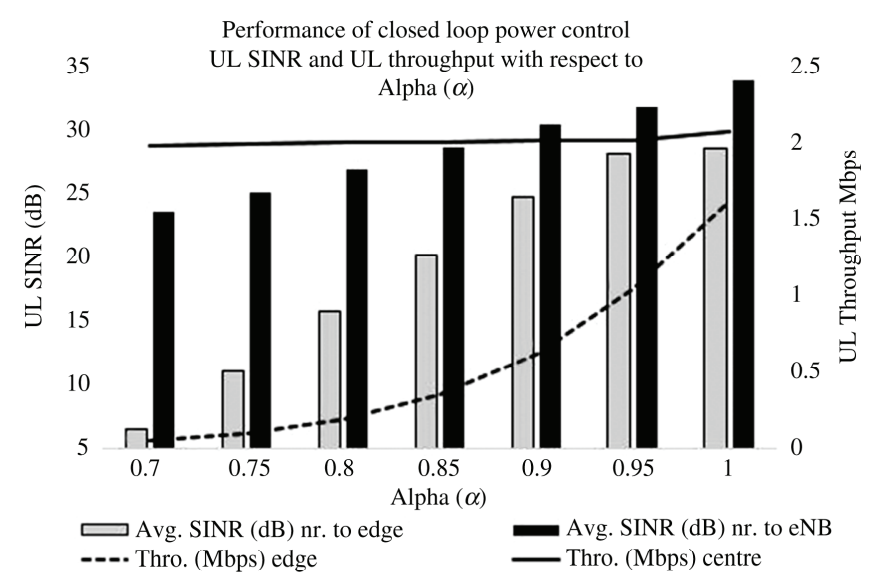

Figure 7. UL throughput for CLUPC with respect to $\alpha$ (compensation factor).

\section{Conclusion}

Power consumption towards the UE is a prime concern as it affects battery consumption. That's why UL power control is a dominating factor that affects system performance and user experience, especially at inter-cell boundaries. As per results, we find that the PSDs at the cell boundary are degraded by $5-6 \mathrm{dBW} / \mathrm{Hz}$ and the UL SINR varies from 6 to $28 \mathrm{~dB}$ at the cell boundary for different values of $\alpha$ (which varies from 0.7 to 1 ). Furthermore, we found that OLUPC is better than CLUPC, especially for UE at a boundary or edge position. On further examination of the CLUPC mechanism, we found the optimum value for $\alpha$ to be 0.95 , as the difference of SINR for that value of $\alpha$ between the centre of the cell and edge boundary is minimum with comparatively high throughput. For better performance of UL radio, it is preferred to choose CLUPC with compensation factor near to 0.95 .

\section{Conflict of Interests}

The authors declare that they have no conflict of interest.

\section{References}

[1] 3GPP, 3gpp, [Online], http://www.3gpp.org/dynareport/36series.htm [Accessed 0102 2017].
[2] X. Wang, F.-C. Zheng, P. Zhu, and X. You, Energy-efficient resource allocation in coordinated downlink multicell OFDMA systems, IEEE Transactions on Vehicular Technology, 65(3), 2015, 1395-1408.

[3] P. Yang, M.D. Renzo, S. Li, and L. Hanzo, Design guidelines for spatial modulation, IEEE Communication Surveys \& Tutorials, 17(1), 2015, 6-26.

[4] J. Parik and S. Mishra, Effect of ISD on pathloss and SINR in 4G systems, IEEE IMPACT-2013, Aligarh, India, 2013, $23-25$.

[5] G. Ku and J.M. Walsh, Resource allocation and link adaptation in LTE and LTE-advanced: A tutorial, IEEE Communications Surveys and Tutorials, 17(3), 2013, 1605-1633.

[6] A. Saxena and R. Sindal, Characterizing the effect of N-CoMP and CoMP with interference in LTE-A, Int. Conf. Computer Communication and Informatics ICCCI 2016, Coimbatore, 2016.

[7] A. Saxena and R. Sindal, Typical effect of CoMP under imperfect CSI in LTE-A, International Conference on Smart Trends for Information Technology and Computer Communications (SmartCom-2016), Vol. 628, Jaipur, Rajasthan, Springer Lecture Notes in CCIS series - Communications in Computer and Information Science, 2016, 509-517.

[8] A. Saxena and R. Sindal, Analysis of cell reselection in evolved umts radio access network radio resource management, Second IEEE Int. Conf. Engineering and Technology (ICETECH), Coimbatore, TN, India, 17th-18th March 2016.

[9] A. Saxena and R. Sindal, Performance Analysis of MAC Scheduler in LTE (EUTRAN) for "ASAR": Resource Allocation, Next Generation Mobile Applications, Services and Technologies (NGMAST 2016), Cardiff, UK, 2016.

[10] A. Saxena and R. Sindal, Strategy for resource allocation in LTE-A, IEEE Int. Conf. Signal Processing, Communication, Power and Embedded System (SCOPES)-2016, Centurion University of Technology and Management (CUTM), Paralakhemundi Campus, Orissa India, 3-5 October 2016.

[11] A. Saxena and R. Sindal, Performance Analysis of Proportional Fair MAC Scheduler under varying feedback delay in EUTRAN, International Journal of Communications, 4(1), 2016, 1-8.

[12] A. Saxena and R. Sindal, Comparative performance analysis of different resource scheduler and characterizing the impact of proportional fair under different TTI feedback delay in EUTRA network, International Journal of Mobile Network Design and Innovation, vol. In Press, pp. X-Y, 2017.

\section{Biographies}

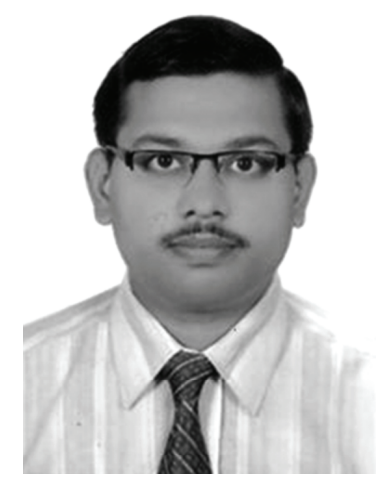

Ankit Saxena holds a Diploma degree in Electronic Communication. He obtained a Bachelor of Engineering degree in Electronics and Instrumentation, a Master's degree in Business Administration (System) and a Master's degree in Electronics Engineering (Electronics and Telecommunication Engineering). He started his career in the telecom industry with a major telecom operator in different technologies: Airtel in 2000, Tata (2003-2004), Reliance (2004-2008); telecom product manufacture and manage services in Alcatel-Lucent (2008-2009) and Ericsson (2009-2015). He is currently working towards the Ph.D. degree in Electronics and Telecommunication in the Laboratory of the Institute of Engineering and Technology, Devi Ahilya University, Indore. His current research is in the area of performance analysis and key performance improvement in EUTRAN network (LTE and LTE-Advance), and reviewer of various International Journals and Conferences. 


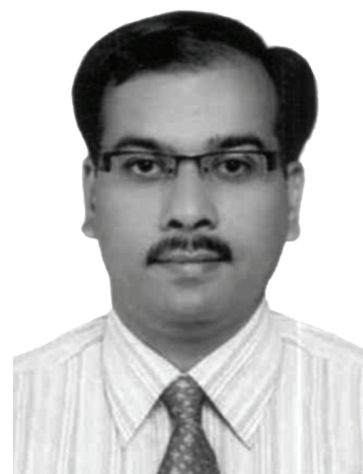

Ravi Sindal received the B.E. (Hons.) degree from Rajasthan University, Jaipur in 1998. He received the M.Tech. Degree, in Instrumentation and Ph.D. degree in Electronics and Telecommunication from Devi Ahilya University in 2000 and 2011, respectively. Since 2013, he has been a Professor of Electronics and Telecommunication at the Institute of Engineering and Technology, Devi Ahilya University, Indore. His research interests include Radio Resource Management, Modeling and Simulation, Digital Design and wireless networks. He has served on the program committee of various conferences. He has published many papers in various well-known International Journals and Conferences. 